\title{
Journal Scan
}

\section{U. C. Ojha}

Senior Specialist \& Head - Department of Respiratory Medicine, E.S.I Hospital, New Delhi

1. Sleep Breath. 2011 Dec;15(4):837-43.

\section{Use of high-frequency peak in spectral analysis of heart rate increment to improve screening of obstructive sleep apnoea.}

\section{Poupard L, Court-Fortune I, Pichot V, Chouchou F, Barthélémy JC, Roche F. Novacor, Rueil Malmaison, France.}

PURPOSE: Several studies have correlated the ratio of the very low frequency power spectral density of heart rate increment (\%VLFI) with obstructive sleep apnoea syndrome (OSAS). However, patients with impaired heart rate variability may exhibit large variations of heart rate increment (HRI) spectral pattern and alter the screening accuracy of the method.

METHODS: To overcome this limitation, the present study uses the high-frequency increment (HFI) peak in the HRI spectrum, which corresponds to the respiratory influence on RR variations over the frequency range 0.2 to $0.4 \mathrm{~Hz}$. We evaluated 288 consecutive patients referred for snoring, observed nocturnal breathing cessation and/ or daytime sleepiness. Patients were classified as OSAS if their apnoea plus hypopnoea index (AHI) during polysomnography exceeded 15 events per hour. Synchronized electrocardiogram Holter monitoring allowed HRI analysis.

RESULTS: Using a \%VLFI threshold $>2.4 \%$ for identifying the presence of OSAS,

\section{Address for correspondence}

Dr. U. C. Ojha

Senior Consultant and Head of the Department Specialist \& Head, Dept of Respiratory Medicine E.S.I. Hospital, Basaidarapur, New Delhi Email: ucojha@rediffmail.com sensitivity for OSAS was $74.9 \%$, specificity $51 \%$, positive predictive value $54.9 \%$ and negative predictive value $71.7 \%$ (33 false negative subjects). Using threshold for $\% \mathrm{VLFI}>2.4 \%$ and $\mathrm{HFI}$ peak position $>0.4 \mathrm{~Hz}$, negative predictive value increased to $78.2 \%$ while maintaining specificity at $50.6 \%$. Among 11 subjects with \%VLFI $<2.4 \%$ and HFI peak $>0.4 \mathrm{~Hz}$, nine demonstrated moderate to severe OSAS (AHI >30).

CONCLUSIONS: HFI represents a minimal physiological criterion for applying \%VLFI by ensuring that heart rate variations are band frequency limited.

\section{Sleep Breath. 2011 May;15(2):221-7.}

\section{Facial patterns and primary nocturnal enuresis in children.}

\section{Carotenuto M, Esposito M, Pascotto A.}

Sleep Center for Developmental Age, Clinic of Child and Adolescent

Neuropsychiatry, Second University of Naples, Via Sergio Pansini n. 5-PAD XI,

80131 Naples, Italy. marco.carotenuto@unina2.it

PURPOSE: Aims of our study are evaluating: (1) the prevalence of dolicofacial pattern among enuretic and control-group children, (2) the prevalence of an abnormal head posture in bedwetters, and (3) the correlation with sleep-related breathing disorders (SRBD) identified by polysomnography (PSG) recording.

Nocturnal enuresis is a multifactorial disease, but has been related also to obstructive sleep-disordered breathing in both adults and children. Anatomical factors recognized to predispose to SRBD include adenotonsillar hypertrophy, neuromuscular disorders, craniofacial abnormalities associated with macroglossia, retrognathia

Indian Journal of Sleep Medicine (IJSM), Vol. 7, No. 3, 2012 
or maxillary hypoplasia, and obesity.

METHODS: Two hundred seventy enuretic children aged $7-12$ years (mean $9.62 \pm 2.31$ ) were compared with a control-matched group of 274 children. To screen nocturnal sleep habits, all subjects' mothers filled out the Sleep Disturbance Scale for Children. Among these scales, only SBD scale was taken into account. Sleep breathing disorders (SBD) scale is composed of three items: sleep breathing difficulties, sleep apnea, and snoring. Cephalic index was calculated for each patient in order to identify facial patterns. An overnight PSG was performed in 28 (15 males, 13 females), randomly chosen, enuretic children and in 38 healthy volunteer controls (18 males, 20 females) matched for age (8.73 $\pm \quad 0.79$ vs. $9.12 \pm 1.23 ; \mathrm{p}=0.147)$ and sex distribution (chi-square $=0.062 ; \mathrm{p}=0.803)$.

RESULTS: Bedwetters show different sleep habits, higher prevalence of dolicofacial pattern, and abnormal head posture more than controls.

CONCLUSIONS: Our preliminary study support further investigation of usage of the cephalic index as a predictor of SRBD.

3. Int J Chron Obstruct Pulmon Dis. 2011;6:551-61.

\section{Use of cluster analysis to describe desaturator phenotypes in COPD: correlations between pulmonary function tests and nocturnal oxygen desaturation.}

Toraldo DM, De Nuccio F, Gaballo A, Nicolardi G.

A Galateo Lung Disease Hospital, Regional Service Puglia, San Cesario di Lecce, Italy. d.torald@tin.it

BACKGROUND: Significant heterogeneity of clinical presentation and disease progression exists within chronic obstructive pulmonary disease (COPD). Although forced expiratory volume in 1 second (FEV(1)) inadequately describes this heterogeneity, a clear alternative has not emerged. This article discusses and refines the concept of phenotyping desaturators in COPD and shows a possible pattern which could be used as a framework for future research.

RECENT FINDINGS: COPD is a complex condition with pulmonary and extrapulmonary manifestations. We suggest that COPD phenotypes should be associated with clinically meaningful outcomes. The innovation of COPD phenotyping is defined as COPD desaturators. Sleep-related hypoxemia and hypercapnia are well recognized in COPD and the development of systemic inflammation during sleep. These sleep-related changes predispose to nocturnal cardiac arrhythmias, pulmonary hypertension, and possibly death, particularly during acute exacerbations.

CONCLUSION: A more focused definition makes possible a classification of patients into two distinct subgroups for both clinical and research purposes. Establishing a common language for future research will facilitate our understanding and management of such diseases. Even if different treatment strategies have different outcomes for these groups, we will have confirmation, or otherwise, of the clinical value of cluster analysis. This knowledge could lead to pharmacological treatment and other interventions directed to specific phenotypic groups.

4. Acta Otorhinolaryngol Ital. 2011 Jun;31(3):149-53.

Diagnostic and therapeutic iter in
paediatric OSAS: personal experience.

Piumetto E, Sammartano AM, Meinardi G, Dagna F, Gervasio FC, Albera R.

Audiology and Phoniatry Clinic, University of Turin, "San Giovanni Battista di

Torino" Hospital, Turin, Italy. elenapiumetto@libero.it Obstructive sleep apnoea syndrome in a child is characterized by prolonged episodes of obstructive hypopnoea and/or apnoea of upper airway leading to morbidity. The most common risk factor is adenotonsillar hypertrophy.

Obstructive sleep apnoea syndrome diagnosis is based on clinical ENT evaluation and an instrumental approach, such as pulse oximetry or the gold standard overnight polysomnography. The aim is to establish, in a population of children with suspected obstructive sleep apnoea syndrome, the frequency of this disorder, the effect of adenotonsillectomy and the risk of postoperative complications. A total of 481 patients (297 male, 184 female) with suspected obstructive sleep apnoea syndrome (aged 2-14 years) were evaluated 
between March 2007 and April 2010 and divided into 3 morphological phenotypes: classic, adult and congenital.

All patients underwent ENT assessment and a pulse oximetry with 4 channels cardiopulmonary monitoring. The examination following the Brouillette criteria was defined as negative, positive or inconclusive; when positive, adenotonsillectomy was the first therapeutic approach. At 6 months after surgery, all patients underwent check-up pulse oximetry. Of the overall sample, $96 \%$ of the patients had a classical phenotype, $3 \%$ an adult type and $1 \%$ a congenital type.

The monitoring resulted pathological in 19\% (17\% of them were at increased post-operative risk), negative in $61 \%$ and inconclusive in $20 \%$. All 5 patients with congenital phenotype were positive. Of the positive patients, 86\% underwent adenotonsillectomy and a control pulse oximetry 6 months thereafter, $96 \%$ resulted negative. Pulse oximetry was efficient in order to avoid incorrect surgery indications, improving appropriateness and safety of adenotonsillectomy in children with obstructive sleep apnoea syndrome. Adenotonsillectomy showed a success rate of $96 \%$ and there were no episodes of post-surgery complications in particular in those patients at increased risk.

5. Sleep. 2011 Jul 1;34(7):957-64.

Heritability and mortality risk of insomnia-related symptoms: a genetic epidemiologic study in a populationbased twin cohort.

\section{Hublin C, Partinen M, Koskenvuo M, Kaprio J.}

Finnish Institute of Occupational Health, Helsinki, Finland.

\section{christer.hublin@ttl.fi}

STUDY OBJECTIVES: Our aim was to estimate heritability in phenotypic insomnia and the association between insomnia and mortality.

DESIGN: Representative follow-up study.

PARTICIPANTS: 1990 survey of the Finnish Twin Cohort ( $\mathrm{N}=12502$ adults; 1554 monozygotic and 2991 dizygotic twin pairs).

MEASUREMENTS: Current insomnia-related symptoms (insomnia in general, difficulty in initiating sleep, sleep latency, nocturnal awakening, early morning awakening, and non-restorative sleep assessed in the morning and during the day) were asked.

Latent class analysis was used to classify subjects into different sleep quality classes. Quantitative genetic modelling was used to estimate heritability.

Mortality data was obtained from national registers until end of April 2009.

RESULTS: The heritability estimates of each symptom were similar in both genders varying from 34\% (early morning awakening) to $45 \%$ (nocturnal awakening). The most parsimonious latent class analysis produced 3 classes: good sleepers (48\%), average sleepers (up to weekly symptoms, $40 \%$ ), and poor sleepers (symptoms daily or almost daily, 12\%). The heritability estimate for the cluster was $46 \%$ (95\% confidence interval $41 \%$ to $50 \%$ ). In a model adjusted for smoking, BMI, and depressive symptoms, the all-cause mortality of poor sleepers was elevated (excess mortality $55 \%$ in men and $51 \%$ in women). Further adjustment for sleep length, use of sleep promoting medications, and sleep apnearelated symptoms did not change the results.

CONCLUSIONS: Insomnia-related symptoms were common in both genders. The symptoms and their clusters showed moderate heritability estimates. A significant association was found between poor sleep and risk of mortality, especially in those with somatic disease.

6. Expert Rev Respir Med. 2011 Jun;5(3):377-93.

\section{Asthma-related comorbidities.}

\section{Boulet LP, Boulay MÈ.}

Centre de Recherche de l'Institut Universitaire de Cardiologie et de Pneumologie de Québec, 2725, Chemin Sainte-Foy, QC, G1V 4G5, Canada. lpboulet@med.ulaval.ca

Asthma is often associated with various comorbidities. The most frequently reported asthma comorbid conditions include rhinitis, sinusitis, gastroesophageal reflux disease, obstructive sleep apnea, hormonal disorders and psychopathologies. These conditions may, first: share a common pathophysiological mechanism with asthma; second: influence asthma control, its phenotype and response to treatment; and third: be more

Indian Journal of Sleep Medicine (IJSM), Vol. 7, No. 3, 2012 
prevalent in asthmatic patients but without obvious influence on this disease. For many of these, how they interact with asthma remains to be further documented, particularly for severe asthma. If considered relevant, they should, however, be treated appropriately. Further research is needed on the relationships between these conditions and asthma.

7. J Sleep Res. 2011 Dec;20(4):526-32.

\section{Phenotyping interindividual variability in obstructive sleep apnoea response to temazepam using ventilatory chemoreflexes during wakefulness.}

\section{Wang D, Marshall NS, Duffin J, Yee BJ, Wong KK, Noori N, Ng SS, Grunstein RR.}

Department of Respiratory and Sleep Medicine, Royal Prince Alfred Hospital, Camperdown, Sydney, NSW, Australia. david.wang@sydney.edu.au

Centrally active agents have a variable impact in patients with obstructive sleep apnoea (OSA) that is unexplained. How to phenotype the individual OSA response is clinically important, as it may help to identify who will be at risk of respiratory depression and who will benefit from a centrally active agent. Based on loop gain theory, we hypothesized that OSA patients with higher central chemosensitivity have higher breathing instability following the use of a hypnosedative, temazepam. In 20 men with OSA in a double-blind, placebo-controlled cross-over trial we tested the polysomnographically (PSG) measured effects of temazepam $10 \mathrm{mg}$ versus placebo on sleep apnoea. Treatment nights were at least 1 week apart. Ventilatory chemoreflexes were also measured during wakefulness in each subject. The patients (mean \pm standard deviation; $44 \pm 12$ years) had predominantly mild-to-moderate OSA [baseline apnoea-hypopnoea index $(\mathrm{AHI})=16.8 \pm 14.1]$. Patients' baseline awake central chemosensitivity correlated significantly with both the change of $\mathrm{SpO}$, nadir between temazepam and placebo $(\mathrm{r}=-0.468, \mathrm{P}=0.038)$ and oxygen desaturation index $(\mathrm{ODI} ; \mathrm{r}=0.485, \mathrm{P}=0.03)$, but not with the change of AHI $(r=0.18, P=0.44)$. Peripheral chemosensitivity and ventilatory recruitment threshold were not correlated with the change of SpO, nadir, ODI or AHI (all P > 0.05). Mild-moderate OSA patients with higher awake central chemosensitivity had greater respiratory impairment during sleep with temazepam. Relatively simple daytime tests of respiratory control may provide a method of determining the effect of sedative-hypnotic medication on breathing during sleep in OSA patients.

8. Eur Arch Otorhinolaryngol. 2012 Jan;269(1):331-7. Epub 2011 May 29.

\section{Obstructive sleep apnea in Treacher Collins syndrome.}

Akre H, Øverland B, Åsten P, Skogedal N, Heimdal K.

Sleep Unit, Department of Otorhinolaryngology-Head and Neck Surgery, Lovisenberg Diakonale Hospital, 0440 Oslo, Norway. haak@lds.no

The aim of the present study was to investigate the prevalence of obstructive sleep apnea syndrome (OSAS) among the Norwegian population with Treacher Collins syndrome (TCS). A secondary aim was to establish whether TCS phenotype severity is associated with OSAS severity. A prospective case study design was used.

Individuals who were 5 years old and above with a known diagnosis of TCS in Norway were invited to participate in a study. The study included genetic testing, medical and dental examinations and polysomnography. All participants demonstrated disturbed respiration during sleep; 18/19 met the diagnostic criteria for OSAS. Subjectively evaluated snoring was not a reliable predictor of OSAS. We found no significant association between TCS phenotype severity and the severity of OSAS. OSAS is common in TCS, but there is no association with the phenotype severity. Individuals diagnosed with TCS must undergo sleep studies to identify the presence of OSAS.

9. Can J Neurol Sci. 2011 May;38(3):411-6.

\section{Hyperekplexia: treatment of a severe phenotype and review of the literature.}

\section{Mineyko A, Whiting S, Graham GE.}

Division of Neurology, Department of Genetics, Children's Hospital of Eastern Ontario, Ottawa, Ontario, Canada. 
Hyperekplexia is a rare disorder caused by autosomal dominant or recessive modes of inheritance and characterized by episodes of exaggerated startle. Five causative genes have been identified to date. The syndrome has been recognized for decades and due to its rarity, the literature contains mostly descriptive reports, many early studies lacking molecular genetic diagnoses. A spectrum of clinical severity exists. Severe cases can lead to neonatal cardiac arrest and death during an episode, an outcome prevented by early diagnosis and clinical vigilance. Large treatment studies are not feasible, so therapeutic measures continue to be empiric. A marked response to clonazepam is often reported but refractory cases exist. Herein we report the clinical course and treatment response of a severely affected infant homozygous for an SLC6A5 nonsense mutation and review the literature summarizing the history and genetic understanding of the disease as well as the described comorbidities and treatment options.

10. Expert Rev Neurother. 2011 Apr;11(4):565-77.

\section{Sleep disturbances in children with attention-deficit/hyperactivity disorder.}

\section{Spruyt K, Gozal D.}

Department of Pediatrics, Comer Children's Hospital, Pritzker School of Medicine, The University of Chicago, Chicago, Illinois 60637, USA.

In this article, we advocate the need for better understanding and treatment of children exhibiting inattentive, hyperactive, impulsive behaviors, by in-depth questioning on sleepiness, sleep-disordered breathing or problematic behaviors at bedtime, during the night and upon awakening, as well as night-to-night sleep duration variability. The relationships between sleep and attentiondeficit/hyperactivity disorder (ADHD) are complex and are routinely overlooked by practitioners. Motricity and somnolence, the most consistent complaints and objectively measured sleep problems in children with ADHD, may develop as a consequence of multidirectional and multifactorial pathways.

Therefore, subjectively perceived or reported restless sleep should be evaluated with specific attention to restless legs syndrome or periodic limb movement disorder, and awakenings should be queried with regard to parasomnias, dyssomnias and sleep-disordered breathing. Sleep hygiene logs detailing sleep onset and offset quantitatively, as well as qualitatively, are required. More studies in children with ADHD are needed to reveal the 24-h phenotype, or its sleep comorbidities.

11.

\section{Residual sleepiness in obstructive sleep apnoea: phenotype and related symptoms.}

\section{Vernet C, Redolfi S, Attali V, Konofal E, Brion A, Frija-Orvoen E, Pottier M, Similowski T, Arnulf I.}

Sleep Disorders Unit, UMR 975, National Reference Centre for Narcolepsy and Hypersomnia, Paris, France.

The characteristics of residual excessive sleepiness (RES), defined by an Epworth score $>10$ in adequately treated apnoeic patients, are unknown. 40 apnoeic patients, with $(\mathrm{n}=20)$ and without $(\mathrm{n}=20)$ RES, and 20 healthy controls underwent clinical interviews, cognitive and biological tests, polysomnography, a multiple sleep latency test, and 24-h sleep monitoring. The marked subjective sleepiness in the RES group (mean \pm sd score $16.4 \pm 3$ ) contrasted with moderately abnormal objective measures of sleepiness (90\% of patients with RES had daytime sleep latencies $>8 \mathrm{~min}$ ). Compared with patients without RES, the patients with RES had more fatigue, lower stage N3 percentages, more periodic leg movements (without arousals), lower mean sleep latencies and longer daytime sleep periods.

Most neuropsychological dimensions (morning headaches, memory complaints, spatial memory, inattention, apathy, depression, anxiety and lack of selfconfidence) were not different between patients with and without RES, but gradually altered from controls to apnoeic patients without and then with RES. RES in apnoeic patients differs markedly from sleepiness in central hypersomnia. The association between RES, periodic leg movements, apathy and depressive mood parallels the post-hypoxic lesions in noradrenaline, dopamine and serotonin systems in animals exposed to intermittent hypoxia.

Indian Journal of Sleep Medicine (IJSM), Vol. 7, No. 3, 2012 


\section{PHOX2B mutations in patients with Ondine-Hirschsprung disease and a review of the literature.}

\section{Kwon MJ, Lee GH, Lee MK, Kim JY, Yoo HS, Ki CS, Chang YS, Kim JW, Park WS.}

Department of Laboratory Medicine, Kangbuk Samsung Hospital, Sungkyunkwan

University School of Medicine, 108 Pyeong-dong, Jongno-gu, Seoul, Republic of Korea 110-746.

Congenital central hypoventilation syndrome (CCHS), also known as Ondine's curse, is characterized by idiopathic failure of autonomic breathing and is often associated with neurocristopathies such as Hirschsprung disease (HSCR). CCHS is caused by mutations in the paired-like homeobox 2B (PHOX2B) gene, often manifest as polyalanine repeat expansions. Herein, we report the cases of two unrelated Korean patients with OndineHirschsprung disease. The patient's clinical manifestations were apnea and cyanosis requiring immediate endotracheal intubation, recurrent hypoventilation with hypercapnia, hypoxia after ventilator removal, and abdominal distension since birth. Intestinal biopsies were performed and the absence of ganglion cells in the colon was consistent with HSCR. We performed direct sequencing analysis in the PHOX2B and RET genes and fluorescence polymerase chain reaction in order to determine the polyalanine tract expansion in exon 3 of the PHOX2B gene. Expansion mutations were detected in both patients; one had 20/ 24 repeats and the other had 20/27 repeats. The 20/24 genotype has not been previously described in severe CCHS phenotypes and associated HSCR. We believe that the information in this report will improve our understanding of the phenotypic and genotypic heterogeneities of CCHS and HSCR.

Indian Journal of Sleep Medicine (IJSM), Vol. 7, No. 3, 2012

\section{Pathophysiology of resistant hypertension: the role of sympathetic nervous system}

\author{
Tsioufis C, Kordalis A, Flessas D, \\ Anastasopoulos I, Tsiachris D, \\ Papademetriou V, Stefanadis C.
}

First Cardiology Clinic, University of Athens, Hippokration Hospital, 3 Kolokotroni Street, P. Penteli, Athens 15236, Greece.

Resistant hypertension (RH) is a powerful risk factor for cardiovascular morbidity and mortality. Among the characteristics of patients with RH, obesity, obstructive sleep apnea, and aldosterone excess are covering a great area of the mosaic of $\mathrm{RH}$ phenotype. Increased sympathetic nervous system (SNS) activity is present in all these underlying conditions, supporting its crucial role in the pathophysiology of antihypertensive treatment resistance. Current clinical and experimental knowledge points towards an impact of several factors on SNS activation, namely, insulin resistance, adipokines, endothelial dysfunction, cyclic intermittent hypoxaemia, aldosterone effects on central nervous system, chemoreceptors, and baroreceptors dysregulation. The further investigation and understanding of the mechanisms leading to SNS activation could reveal novel therapeutic targets and expand our treatment options in the challenging management of $\mathrm{RH}$.

14. Sleep. $2011 \mathrm{Feb}$ 1;34(2):165-70.

\section{Daytime sleepiness and REM sleep characteristics in myotonic dystrophy: a case-control study.}

\section{Yu H, Laberge L, Jaussent I, Bayard S, Scholtz S, Raoul M, Pages M, Dauvilliers Y.}

Service de Neurologie, Hôpital Gui-de-Chauliac, 80 avenue Augustin Fliche, Montpellier cedex 5, France.

STUDY OBJECTIVES: Excessive daytime sleepiness (EDS) and high daytime REM sleep pressure are important sleep features of myotonic dystrophy (DM1). Small and uncontrolled studies have focused on EDS phenotype; none have focused on nocturnal REM sleep 
characteristics in DM1. Our objectives were to compare polysomnographic and multiple sleep latency test (MSLT) parameters, and both tonic and phasic components of REM sleep between DM1 and controls.

DESIGN AND PATIENTS: Forty consecutive DM1 patients and 40 sex- and age-matched controls were included. All subjects underwent overnight polysomnography followed by a MSLT.

RESULTS: About $80 \%$ of DM1 patients complained of EDS through clinical interview: $31.4 \%$ had Epworth scores $>10$, and $12.5 \%$ had objective sleepiness (latency $<8 \mathrm{~min}$ ). Higher apnea and central apnea indexes, and a greater proportion of subjects with severe apnea/ hypopnea syndrome were found in DM1. The number of SOREMP differed between DM1 and controls, one and two SOREMPs being present in $47.5 \%$ and $32.5 \%$, and one control had one SOREMP. Higher percentages of slow wave sleep and REM sleep were found in DM1. DM1 patients had significantly more PLMW, PLMS in both NREM and REM sleep, and PLMS-associated microarousals. Higher REM density was found in DM1 with similar tendencies for either REM sleep without atonia or phasic EMG activity.

CONCLUSIONS: This is the first case-control sleep study in DM1 to demonstrate higher frequency of daytime sleepiness and abnormalities in REM sleep regulation, with an increased daytime and nighttime REM sleep propensity, REM density, and PLMS. These data suggest a primary central sleep regulation dysfunction in DM1.

15. J Korean Med Sci. 2011 Feb;26(2):312-5. Epub 2011 Jan 24.

\section{Haddad syndrome with PHOX2B gene mutation in a Korean infant.}

\section{Lee CW, Lee JH, Jung EY, Choi SO, Kim CS Lee SL, Kim DK.}

Institute for Medical Genetics, Keimyung University College of Medicine, Daegu, Korea.

Congenital central hypoventilation syndrome with Hirschsprung's disease, also known as Haddad syndrome, is an extremely rare disorder with variable symptoms.

Recent studies described that congenital central hypoventilation syndrome had deep relation to the mutation of the PHOX2B gene in its diagnosis and phenotype.

We report a newborn male infant with clinical manifestations of recurrent hypoventilation with hypercapnea and bowel obstruction. These clinical manifestations were compatible with congenital central hypoventilation syndrome and Hirschsprung's disease, and polyalanine 26 repeats in the PHOX2B gene supported the diagnosis of congenital central hypoventilation. We described a first case of Haddad syndrome in Korean and its clinical and genetic characteristics were discussed.

\section{Pac Symp Biocomput. 2011:14-25.}

\section{Systems biology analyses of gene expression and genome wide association study data in obstructive sleep apnea.}

\section{Liu Y, Patel S, Nibbe R, Maxwell S, Chowdhury SA, Koyuturk M, Zhu X, Larkin EK,}

\section{Buxbaum SG, Punjabi NM, Gharib SA, Redline S, Chance MR.}

Center for Proteomics \& Bioinformatics, Case Western Reserve University (CWRU), Cleveland, Ohio, 44106, USA.yx1442@case.edu.

The precise molecular etiology of obstructive sleep apnea (OSA) is unknown; however recent research indicates that several interconnected aberrant pathways and molecular abnormalities are contributors to OSA. Identifying the genes and pathways associated with OSA can help to expand our understanding of the risk factors for the disease as well as provide new avenues for potential treatment.

Towards these goals, we have integrated relevant high dimensional data from various sources, such as genomewide expression data (microarray), protein-protein interaction (PPI) data and results from genome-wide association studies (GWAS) in order to define subnetwork elements that connect some of the known pathways related to the disease as well as define novel regulatory modules related to OSA. Two distinct approaches are applied to identify sub-networks 
significantly associated with OSA. In the first case we used a biased approach based on sixty genes/proteins with known associations with sleep disorders and/or metabolic disease to seed a search using commercial software to discover networks associated with disease followed by information theoretic (mutual information) scoring of the sub-networks. In the second case we used an unbiased approach and generated an interactome constructed from publicly available gene expression profiles and PPI databases, followed by scoring of the network with p-values from GWAS data derived from OSA patients to uncover sub-networks significant for the disease phenotype. A comparison of the approaches reveals a number of proteins that have been previously known to be associated with OSA or sleep. In addition, our results indicate a novel association of Phosphoinositide 3-kinase, the STAT family of proteins and its related pathways with OSA.

17. Ann Hematol. 2011 Feb;90(2):145-50.

\section{Cost-effectiveness of adenotonsillectomy in reducing obstructive sleep apnea, cerebrovascular ischemia, vaso-occlusive pain, and ACS episodes in pediatric sickle cell disease.}

\section{Tripathi A, Jerrell JM, Stallworth JR.}

Department of Epidemiology and Biostatistics, University of South Carolina Arnold School of Public Health, Columbia, SC, USA.

In children with sickle cell disease (SCD), adenotonsillar hypertrophy or recurrent tonsillitis are frequently linked with an increased risk of obstructive sleep apnea, cerebrovascular ischemia, or frequent pain episodes and often require an adenoidectomy and/or tonsillectomy. Interventions designed to prevent these complications, control vaso-occlusive pain episodes, and avoid hospitalizations may reduce the significant personal and economic burden of SCD.
This study compares episode recurrence and treatment costs for cerebrovascular ischemia, vaso-occlusive pain, acute chest syndrome (ACS), and obstructive sleep apnea in children who had an adenotonsillectomy (A/T surgery, $\mathrm{N}=256 ; 11.7 \%)$ and a matched cohort of those who did not $(\mathrm{N}=512 ; 23.3 \%)$ from a cohort of 2,194 children and adolescents with SCD from South Carolina's Medicaid system. A/T surgery was associated with a significantly reduced rate of visits over time for obstructive sleep apnea and cerebrovascular ischemia (e.g., stroke, transient ischemic attacks), but not with any change in the rate of visits for vaso-occlusive pain or ACS/pneumonia visits. The rate of mean acute (emergency and inpatient) service costs was significantly decreasing over time after an increase about the time the $\mathrm{A} / \mathrm{T}$ surgery was performed. The cost-effectiveness of adenoidectomy and/or tonsillectomy for treating obstructive sleep apnea and preventing cerebrovascular ischemia without increasing vaso-occlusive pain episodes or long-term acute service costs in routine clinical practice settings was demonstrated. The matched control group of SCD patients without A/T surgery contained more patients with severe vaso-occlusive pain episodes, ACS visits, and higher mean total costs over time and appears to represent a different phenotype of children with SCD. 